\title{
Nonemptiness Problems of Wang Cubes with Two Colors
}

\author{
Hung-Hsun Chen, Wen-Guei Hu* and Song-Sun Lin
}

\begin{abstract}
This investigation studies the nonemptiness problems of Wang cubes with two colors. Wang cubes are unit cubes with colored faces, which are generalized from Wang tiles. For a set $B$ of Wang cubs, $\Sigma(B)$ is the set of all global patterns on $\mathbb{Z}^{3}$ that can be constructed by the cubes in $B$. The nonemptiness problem is to determine whether $\Sigma(B) \neq \emptyset$ or not. Denote by $\mathcal{P}(B)$ the set of all periodic patterns on $\mathbb{Z}^{3}$ that can be constructed by the cubes in $B$. For Wang cubes, the corresponding Wang's conjecture is that if $\Sigma(B) \neq \emptyset$, then $\mathcal{P}(B) \neq \emptyset$.

We introduce the transition matrices and trace operators to determine whether $\Sigma(B) \neq \emptyset$ and $\mathcal{P}(B) \neq \emptyset$ or not, respectively. A basic set $B$ is called a minimal cycle generator if $\mathcal{P}(B) \neq \emptyset$ but $\mathcal{P}\left(B^{\prime}\right)=\emptyset$ for all $B^{\prime} \varsubsetneqq B$. By computer computation, there exist 86 equivalence classes of minimal cycle generators with two colors. By verifying that the basic sets $B$ that contains no minimal cycle generators satisfy $\Sigma(B)=\emptyset$, we prove that the Wang's conjecture for Wang cubes with two colors is true.
\end{abstract}

\section{Introduction}

Firstly, we briefly review the nonemptiness problems on $\mathbb{Z}^{2}$. The edge coloring problem on $\mathbb{Z}^{2}$ is closely related to lattice models in the scientific modeling of spatial structure. Relevant investigations have been performed on phase transitions; see Baxter [3, 4], Lieb 16, 17 and Penrose 18.

In 1961, in studying proving theorem by pattern recognition, Wang 20] started to study the square tiling of a plane. Wang tiles are unit squares with colored edges. In tiling the infinite Euclidean plane, Wang tiles are arranged side by side on $\mathbb{Z}^{2}$ such that the touching edges of the adjacent tiles have the same colors; the tiles cannot be rotated or reflected. Let $\mathcal{S}_{p}=\{0,1, \ldots, p-1\}$ be a set of $p$ colors, $p \geq 2$. The set of all Wang tiles with $p$ colors is denoted by $W_{2 ; p}$. A finite set $B \subseteq W_{2 ; p}$ of Wang tiles is called a

Received September 18, 2018; Accepted March 19, 2020.

Communicated by Cheng-Hsiung Hsu.

2010 Mathematics Subject Classification. 37B50, 05B45, 52C22, 52C23.

Key words and phrases. nonemptiness problem, Wang cube, Wang's conjecture, aperiodic set, Wang tile. The second author was supported by the National Natural Science Foundation of China under Grant No. 11601355 .

The third author would like to thank the Ministry of Science and Technology, Taiwan (Contract No. MOST 105-2811-M-009-068) for partially supporting this research.

*Corresponding author. 
basic set (of Wang tiles). We call $U=\left(u_{i, j}\right) \in W_{2 ; p}^{\mathbb{Z}^{2}}$ a global pattern (tiling) on $\mathbb{Z}^{2}$ if the continuous edges of the adjacent tiles have the same colors. Let $\Sigma(B)$ be the set of all global patterns that can be constructed from the Wang tiles in $B$. For $m, n \geq 1$, a global pattern $U=\left(u_{i, j}\right) \in W_{2 ; p}^{\mathbb{Z}^{2}}$ is called (doubly) periodic pattern with horizontal period $m$ and vertical period $n$ if

$$
u_{i+m, j}=u_{i, j+n}=u_{i, j}
$$

for all $i, j \in \mathbb{Z}$. Let $\mathcal{P}(B)$ be the set of all periodic patterns that can be constructed from the Wang tiles in $B$. Clearly, $\mathcal{P}(B) \subseteq \Sigma(B)$.

Given a basic set $B$, the nonemptiness problem is to determine whether or not $\Sigma(B) \neq$ $\emptyset$. In [20], Wang conjectured that

$$
\text { if } \Sigma(B) \neq \emptyset \text {, then } \mathcal{P}(B) \neq \emptyset \text {, }
$$

that is, any set of Wang tiles that can tile a plane can tile the plane periodically. If Wang's conjecture (1.1) holds, then the nonemptiness problem can be reduced to determining whether or not $\mathcal{P}(B) \neq \emptyset$.

However, Berger [5] proved that Wang's conjecture was wrong and the nonemptiness problem of Wang tiles is undecidable. He presented a set $B$ of Wang tiles that could only tile the plane aperiodically, that is,

$$
\Sigma(B) \neq \emptyset \quad \text { and } \quad \mathcal{P}(B)=\emptyset
$$

A set $B$ is called an aperiodic set if $B$ satisfies $(1.2)$. Thereafter, the smaller aperiodic sets are found by Robinson, Penrose, Culik and Kari [8, 10, 14, 18, 19]. For $p=2$ and $p=3$, Chen et al. 6, 12 showed that Wang's conjecture (1.2) holds. Recently, the minimal aperiodic set of 11 Wang tiles with four colors was constructed by Jeandal and Rao [13], in the sense that no aperiodic set has less than four colors or less than 11 tiles.

Considering the tiles that are unit squares with colored vertices, currently, the smallest number of colors of aperiodic sets is six [15]. Our previous work 12 showed the Wang's conjecture holds for $p=2$. To find periodic patterns in corner coloring needs much more computation than that in edge coloring. Therefore, the problem for $p=3$ is still under investigation.

In many situations, the three-dimensional problems are more related to our real world phenomena. The three-dimensional face coloring is considered. Wang tiles were generalized to Wang cubes, unite cube with colored faces, by Culik and Kari [9]. In the face coloring of a space, Wang cubes stack the whole space such that the touching faces of adjacent cubes have the same colors. Before stating the main results, some notation is introduced. 
The set of all Wang cubes with $p$ colors is denoted by $W_{3 ; p}$. Let $B \subseteq W_{3 ; p}$ be a set of Wang cubes. Given $m, n, k \geq 1$, let

$$
\mathbb{Z}_{m \times n \times k}=\left\{\left(i_{1}, i_{2}, i_{3}\right) \in \mathbb{Z}^{3} \mid 0 \leq i_{1} \leq m-1,0 \leq i_{2} \leq n-1,0 \leq i_{3} \leq k-1\right\} .
$$

For $m, n, k \geq 1$, we call $U_{m \times n \times k} \in W_{3 ; p}^{\mathbb{Z}_{m \times n \times k}}$ a local pattern on $\mathbb{Z}_{m \times n \times k}$ if the continuous faces of the adjacent cubes have the same colors. Let $\Sigma_{m \times n \times k}(B)$ be the set of all local patterns on $\mathbb{Z}_{m \times n \times k}$ that can be constructed by the Wang cubes in $B$. Denote by $\Gamma_{m \times n \times k}(B)$ the cardinality of $\Sigma_{m \times n \times k}(B)$.

Similarly, we call $U=\left(u_{i_{1}, i_{2}, i_{3}}\right) \in W_{3 ; p}^{\mathbb{Z}^{3}}$ a global pattern (tilling) on $\mathbb{Z}^{3}$ if the continuous faces of the adjacent cubes have the same colors. Denote by $\Sigma(B)$ the set of all global patterns that can be constructed by the Wang cubes in B. Clearly,

$$
\text { if } \Sigma_{m \times n \times k}(B)=\emptyset \text { for some } m, n, k \geq 1 \text {, then } \Sigma(B)=\emptyset \text {. }
$$

Subsequently, periodic patterns on $\mathbb{Z}^{3}$ are introduced. Let $\mathcal{L}_{3}$ be the set of finite index subgroups of $\mathbb{Z}^{3}$. By Hermite normal form, $\mathcal{L}_{3}$ can be parameterized as

$\mathcal{L}_{3}=\left\{\left[\begin{array}{ccc}a_{1} & b_{12} & b_{13} \\ 0 & a_{2} & b_{23} \\ 0 & 0 & a_{3}\end{array}\right] \mathbb{Z}^{3} \mid a_{i} \geq 1\right.$ for $1 \leq i \leq 3$ and $0 \leq b_{i j} \leq a_{i}-1$ for $\left.i+1 \leq j \leq 3\right\}$.

For any sublattice $L \in \mathcal{L}_{3}$, a global pattern $U=\left(u_{i_{1}, i_{2}, i_{3}}\right) \in W_{3 ; p}^{\mathbb{Z}^{3}}$ is called (triply) $L$ periodic if for any $\left(i_{1}, i_{2}, i_{3}\right) \in \mathbb{Z}^{3}$,

$$
u_{i_{1}+l_{1}, i_{2}+l_{2}, i_{3}+l_{3}}=u_{i_{1}, i_{2}, i_{3}}
$$

for all $\left(l_{1}, l_{2}, l_{3}\right) \in L$. In particular, if a global pattern $U$ is $L$-periodic with $b_{12}=b_{13}=$ $b_{23}=0, U$ is called cuboid periodic with period $\left(a_{1}, a_{2}, a_{3}\right)$. Otherwise, $U$ is called parallelopiped periodic.

For $B \subseteq W_{3 ; p}$, let $\mathcal{P}(B)$ be the set of all $L$-periodic patterns that can be constructed from the Wang cubes in $B$. For $m, n, k \geq 1$, let $\mathcal{P}_{m \times n \times k}(B)$ be the set of all cuboid periodic patterns with period $(m, n, k)$ that can be constructed by the cubes in $B$. Notably, every $L$-periodic pattern can be represented as a cuboid periodic pattern with period $(m, n, k)$ for some $m, n, k \geq 1$, that is,

$$
\mathcal{P}(B)=\bigcup_{m, n, k \geq 1} \mathcal{P}_{m \times n \times k}(B)
$$

Denote by $P_{m \times n \times k}(B)$ the cardinality of $\mathcal{P}_{m \times n \times k}(B)$. It is clear that the number $m \cdot n \cdot k$ is greater than or equal to the index $\left|\mathbb{Z}^{3} / L\right|$. Therefore, for determining $\mathcal{P}(B) \neq \emptyset$ in 
computing, to consider general $L$-periodic patterns is much efficient in some cases; see Example 3.4 .

In general, let $Q$ be a $3 \times 3$ integer matrix with $r=\operatorname{rank}(Q) \geq 1$. Clearly, if $1 \leq r \leq 2$, $Q \mathbb{Z}^{3}$ is a subgroup of $\mathbb{Z}^{3}$ with infinite index. For $1 \leq r \leq 3$, a global pattern $U=\left(u_{i_{1}, i_{2}, i_{3}}\right)$ is called $r$-directional periodic if for any $\left(i_{1}, i_{2}, i_{3}\right) \in \mathbb{Z}^{3}$,

$$
u_{i_{1}+l_{1}, i_{2}+l_{2}, i_{3}+l_{3}}=u_{i_{1}, i_{2}, i_{3}}
$$

for all $\left(l_{1}, l_{2}, l_{3}\right)$ in some $Q \mathbb{Z}^{3}$ with $\operatorname{rank}(Q)=r$. For $B \subseteq W_{3 ; p}$, denote by $\mathcal{P}_{r}(B)$ the set of all $r$-directional periodic patterns that can be constructed from the cubes in $B$. Notably,

$$
\Sigma(B) \supseteq \mathcal{P}_{1}(B) \supseteq \mathcal{P}_{2}(B) \supseteq \mathcal{P}_{3}(B)=\mathcal{P}(B)
$$

Now, the Wang's conjecture can be generalized to Wang cubes: for any $B \subseteq W_{3 ; p}$,

$$
\text { if } \Sigma(B) \neq \emptyset \text {, then } \mathcal{P}(B) \neq \emptyset \text {. }
$$

The aperiodic set of Wang tiles in [13] can be easily used to construct a set $B$ of 11 Wang cubes such that Wang's conjecture (1.4) fails with 4 colors, i.e., $\Sigma(B) \neq \emptyset$ and $\mathcal{P}(B)=\emptyset$. Moreover, Culik and Kari [9] constructed a strongly aperiodic set $B^{\prime}$ of 21 Wang cubes with 7 colors such that $\Sigma\left(B^{\prime}\right) \neq \emptyset$ and $\mathcal{P}_{1}\left(B^{\prime}\right)=\emptyset$.

In this work, we prove that Wang's conjecture (1.4) for Wang cubes holds for $p=2$ using a computer. Concerning the number of colors such that (1.4) holds, it only remains open for $p=3$.

As in our previous work 6,12 , the main strategy of the proof is as follows. First, $B$ is called a cycle generator if $\mathcal{P}(B) \neq \emptyset$; otherwise, $B$ is called a non-cycle generator. Moreover, $B$ is called a minimal cycle generator (MCG) if $B$ is a cycle generator and $\mathcal{P}\left(B^{\prime}\right)=\emptyset$ whenever $B^{\prime} \subsetneq B ; B$ is called a maximal non-cycle generator (MNCG) if $B$ is a non-cycle generator and $\mathcal{P}\left(B^{\prime \prime}\right) \neq \emptyset$ for any $B^{\prime \prime} \supsetneqq B$.

For a set $B$ of Wang tiles with two colors, our previous work [7] showed that the positivity of the spatial entropy of $\Sigma(B)$ is completely determined by the minimal cycle generators in $B$. The problem for Wang cubes with two colors is still under investigation.

Given $p \geq 2$, denote the set of all minimal cycle generators by $\mathcal{C}(p)$ and the set of maximal non-cycle generators by $\mathcal{N}(p)$. Clearly,

$$
\mathcal{C}(p) \cap \mathcal{N}(p)=\emptyset
$$

Therefore, Wang's conjecture (1.4) holds for $p \geq 2$ if

$$
\Sigma(B)=\emptyset \text { for any } B \in \mathcal{N}(p)
$$

can be shown. 
For $p=2$, after the symmetry group $O_{h}$ of a unit cube and the permutation group $S_{2}$ of colors of $x$-, $y$ - and $z$-directional faces, respectively, are applied, it is shown that $\mathcal{C}(2)$ has 86 equivalence classes and $\mathcal{N}(2)$ has 28 equivalence classes. Furthermore, (1.4) holds for $p=2$. Indeed, if $\Sigma(B)=\emptyset$, then there exists a basic set $B^{\prime}$ that is in the equivalence class of $B$ such that $\Sigma_{4 \times 3 \times k}\left(B^{\prime}\right)=\emptyset$ for some $k \geq 1$.

Since the period of a periodic pattern for $p=3$ can be much larger than that for $p=2$, the process in this paper cannot work for $p=3$ immediately. To study the Wang's conjecture for $p=3$, it will need more efficient arguments and computer programs to find $\mathcal{C}(3)$ and $\mathcal{N}(3)$.

The rest of the paper is arranged as follows. Section 2 introduces the transition matrices and trace operators. They are very useful in determining whether $\Sigma_{m \times n \times k}(B) \neq$ $\emptyset$ and $\mathcal{P}(B) \neq \emptyset$ or not. Section 3 will introduce the procedure for determining the sets $\mathcal{C}(2)$ and $\mathcal{N}(2)$, and will prove the main result.

\section{Preliminaries}

This section introduces necessary elements in proving the main result, including the symmetries of Wang cubes, the transition matrices and trace operators.

\subsection{Symmetries}

The symmetry of the unit cube is introduced. The symmetry group $O_{h}$ of unit cube has 24 rotation elements and 24 reflection elements, which is isomorphic to $S_{4} \times C(2)$, where $S_{4}$ is the permutation group of order 24 and $C(2)$ is the cyclic group of order 2 . Therefore, given a basic set $B \subseteq W_{3 ; p}$ and any element $\tau \in O_{h}$, another basic set $(B)_{\tau}$ can be obtained by transforming the local patterns in $B$ by $\tau$.

In face coloring, the permutations of colors in the $x$-, $y$ - and $z$-directions are mutually independent. Denote the permutations of colors in the faces in the $x$-, $y$ - and $z$-directions by $\eta_{x} \in S_{p}, \eta_{y} \in S_{p}$ and $\eta_{z} \in S_{p}$, respectively. Now, for any $B \subseteq W_{3 ; p}$, define the equivalence class $[B]$ of $B$ by

$$
[B]=\left\{B^{\prime} \subseteq W_{3 ; p}: B^{\prime}=\left(\left(\left((B)_{\tau}\right)_{\eta_{x}}\right)_{\eta_{y}}\right)_{\eta_{z}}, \tau \in O_{h} \text { and } \eta_{x}, \eta_{y}, \eta_{z} \in S_{p}\right\}
$$

As in 6, 12, whether or not $\Sigma(B) \neq \emptyset$ and $\mathcal{P}(B) \neq \emptyset$ is shown to be independent of the choice of elements in $[B]$. Indeed, for any $B^{\prime} \in[B]$,

$$
\Sigma\left(B^{\prime}\right) \neq \emptyset \quad\left(\text { or } \mathcal{P}\left(B^{\prime}\right) \neq \emptyset\right) \quad \text { if and only if } \quad \Sigma(B) \neq \emptyset \quad(\text { or } \mathcal{P}(B) \neq \emptyset) .
$$

Moreover, for $B^{\prime} \in[B], B^{\prime}$ is an MCG (MNCG) if and only if $B$ is an MCG (MNCG). Therefore, groups $O_{h}$ and $S_{2}$ can be used efficiently to reduce the number of cases $B \subseteq W_{3 ; p}$ that must be considered, and then can greatly reduce the computation time. 


\subsection{Transition matrix}

This subsection introduces the transition matrices for face coloring.

Now, the case $p=2$ is considered. Let $u$ be a unit cube. The face in the positive (negative) $x$-direction is denoted by $x^{+}\left(x^{-}\right)$; the face in the positive (negative) $y$-direction denoted by $y^{+}\left(y^{-}\right)$; the face in the positive (negative) $z$-direction is denoted by $z^{+}\left(z^{-}\right)$; see Figure 2.1. The colors on the six faces of a Wang cube $u$ is denoted by

$$
\left(\begin{array}{lll}
x^{+} & y^{+} & z^{+} \\
x^{-} & y^{-} & z^{-}
\end{array}\right)=\left(\begin{array}{lll}
x^{+}(u) & y^{+}(u) & z^{+}(u) \\
x^{-}(u) & y^{-}(u) & z^{-}(u)
\end{array}\right) .
$$

Moreover, for a local pattern $U_{m \times n \times k}=\left(u_{i_{1}, i_{2}, i_{3}}\right)$ on $\mathbb{Z}_{m \times n \times k}, m, n, k \geq 1$, the color of the face $\alpha^{\beta}$ on the Wang cube $u_{i_{1}, i_{2}, i_{3}}$ is denoted by $\alpha^{\beta}\left(u_{i_{1}, i_{2}, i_{3}}\right)$, where $\alpha$ is $x, y$ or $z$, $\beta=+,-$.

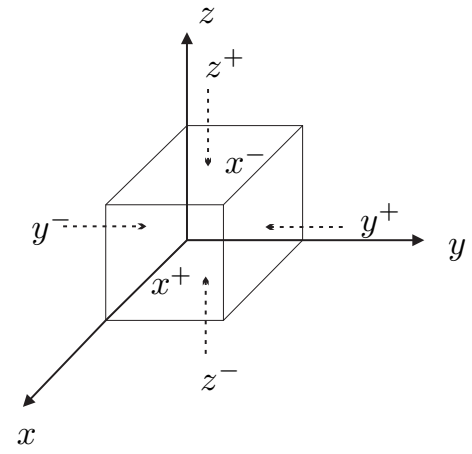

Figure 2.1: The six faces of a unit cube.

Definition 2.1. The coding function $\phi \equiv \phi_{m \times n}:\{0,1\}^{\mathbb{Z}_{m \times n}} \rightarrow\left\{j \mid 0 \leq j \leq 2^{m n-1}\right\}$ is defined as

$$
\phi\left(\left(\beta_{i, j}\right)_{\substack{0 \leq i \leq m-1 \\ 0 \leq j \leq n-1}}\right)=\sum_{i=0}^{m-1} \sum_{j=0}^{n-1} \beta_{i, j} 2^{i+m j} .
$$

Clearly, it can be verified that the coding function $\phi$ is bijective.

Now, given $B \subseteq W_{3 ; 2}$, for computing $\Sigma_{m \times n \times k}(B), m, n, k \geq 1$, the $2^{m n} \times 2^{m n}$ transition matrix $\mathbf{V}_{m \times n}(B)=\left[v_{i, j}(B)\right]_{0 \leq i, j \leq 2^{m n}-1}$ is defined as follows. For $0 \leq i, j \leq 2^{m n}-1$, $v_{i, j}(B)$ is the number of the local patterns $U_{m \times n \times 1}=\left(u_{i_{1}, i_{2}, 0}\right)$ in $\Sigma_{m \times n \times 1}(B)$ such that

$$
i=\phi\left(\left(z^{-}\left(u_{i_{1}, i_{2}, 0}\right)\right)_{\substack{0 \leq i_{1} \leq m-1 \\ 0 \leq i_{2} \leq n-1}}\right) \quad \text { and } \quad j=\phi\left(\left(z^{+}\left(u_{i_{1}, i_{2}, 0}\right)\right)_{\substack{0 \leq i_{1} \leq m-1 \\ 0 \leq i_{2} \leq n-1}}\right) \text {. }
$$

Notably, $i$ determines the colors of top surface of the local patterns $U_{m \times n \times 1}$, and $j$ determines the colors of bottom surface of $U_{m \times n \times 1}$. 
Denote by $|\mathbf{A}|$ the sum of all the entries of a matrix $\mathbf{A}$. Notably, $\left|\mathbf{V}_{m \times n}\right|$ is the number of the local patterns on $\mathbb{Z}_{m \times n \times 1}$ that can be generated by $B$. Furthermore, from the definition of $\mathbf{V}_{m \times n}(B)$, it can be easily verified that, for $m, n, k \geq 1$,

$$
\Gamma_{m \times n \times k}(B)=\left|\mathbf{V}_{m \times n}^{k}(B)\right|
$$

\subsection{Periodic patterns and trace operator}

This subsection studies the trace operator and generalized trace operator for studying periodic patterns of face coloring.

As in [1,2,11], $P_{m \times n \times k}(B)$ can be computed by trace operators as follows. Given $B \subseteq W_{3 ; 2}$, the $2^{m n} \times 2^{m n}$ trace operator $\mathbf{T}_{m \times n}(B)=\left[t_{i, j}(B)\right]_{0 \leq i, j \leq 2^{m n}-1}$ is defined as follows. For $0 \leq i, j \leq 2^{m n}-1, t_{i, j}(B)$ is the number of the local patterns $U_{(m+1) \times(n+1) \times 1}=$ $\left(u_{i_{1}, i_{2}, 0}\right)$ in $\Sigma_{(m+1) \times(n+1) \times 1}(B)$ that satisfy

$$
u_{m, i_{2}, 0}=u_{0, i_{2}, 0} \quad \text { and } \quad u_{i_{1}, n, 0}=u_{i_{1}, 0,0}
$$

for $0 \leq i_{1} \leq m$ and $0 \leq i_{2} \leq n$, and $(2.2)$. Notably, $\left|\mathbf{T}_{m \times n}(B)\right|$ is the number of the local patterns with $x$-period $m, y$-period $n$ and height 1 that can be constructed by the cubes in $B$. The following proposition indicates that the number of cuboid periodic patterns can be computed using the trace of trace operator.

Proposition 2.2. Given $B \subseteq W_{3 ; 2}$, for $m, n, k \geq 1$,

$$
P_{m \times n \times k}(B)=\operatorname{tr}\left(\mathbf{T}_{m \times n}^{k}(B)\right) .
$$

Proof. From the construction of $\mathbf{T}_{m \times n}(B)$, it is clear that $\operatorname{tr}\left(\mathbf{T}_{m \times n}^{k}(B)\right)$ is the number of the local patterns with $x$-period $m, y$-period $n$ and height $k$ that can be constructed by the tiles in $B$ such that the colors of top surface are equal to those of bottom surface. Every such local pattern corresponds uniquely with a cuboid periodic pattern with period $(m, n, k)$. Therefore, (2.4) follows. The proof is complete.

By using $L$-period to present a cuboid periodic pattern with period $(m, n, k)$, the index $\left|\mathbb{Z}^{3} / L\right|=a_{1} \cdot a_{2} \cdot a_{3}$ is always smaller than or equal to $m \cdot n \cdot k$. Indeed, $a_{1} \cdot a_{2} \cdot a_{3}$ is an aliquot part of $m \cdot n \cdot k$. Now, we introduce the generalized trace operator to help us compute the number of certain $L$-periodic patterns.

Given $B \subseteq W_{3 ; 2}$, for $0 \leq q \leq m-1$, the $2^{m n} \times 2^{m n}$ generalized trace operator $\mathbf{T}_{m \times n ; q}=$ $\left[t_{q ; i, j}(B)\right]_{0 \leq i, j \leq 2^{m n}-1}$ is defined as follows.

For $0 \leq i, j \leq 2^{m n}-1, t_{q ; i, j}(B)$ is the number of the local patterns $U_{(m+1) \times(n+1) \times 1}=$ $\left(u_{i_{1}, i_{2}, 0}\right)$ in $\Sigma_{(m+1) \times(n+1) \times 1}(B)$ such that

$$
u_{m, i_{2}, 0}=u_{0, i_{2}, 0} \quad \text { and } \quad u_{i_{1}, n, 0}=u_{\bmod \left(i_{1}+m-q, m\right), 0,0}
$$


for $0 \leq i_{1} \leq m$ and $0 \leq i_{2} \leq n$, and 2.2 . In particular, $\mathbf{T}_{m \times n ; 0}=\mathbf{T}_{m \times n}$. Here, $\bmod (a, b)$ is the remainder when $a$ is divided by $b$. Notably, $\left|\mathbf{T}_{m \times n ; q}(B)\right|$ is the number of the local patterns with two-dimensional parallelogram period with $L^{\prime}=\left[\begin{array}{cc}m & q \\ 0 & n\end{array}\right] \mathbb{Z}^{2}$ and height 1 that can be constructed by the cubes in $B$.

The following proposition is a generalization of Proposition 2.2 .

Proposition 2.3. Given $B \subseteq W_{3 ; 2}$, for $m, n, k \geq 1$ and $0 \leq q \leq m-1$, let $L=\left[\begin{array}{ccc}m & q & 0 \\ 0 & n & 0 \\ 0 & 0 & k\end{array}\right] \mathbb{Z}^{3}$. Then, the number of L-periodic patterns that can be constructed by the cubes in $B$ is $\operatorname{tr}\left(\mathbf{T}_{m \times n ; q}^{k}\right)$.

Proof. Similar to the proof of Proposition 2.2, from the construction of $\mathbf{T}_{m \times n ; q}(B)$, the result follows immediately. The proof is complete.

Notably, using generalized trace operators to find a periodic pattern is more efficient than only using trace operators; see Example 3.4 .

Now, recall some notation and results of matrix theory. A matrix $\mathbf{A}$ is called nilpotent if $\mathbf{A}^{k}$ equals a zero matrix for some $k \geq 1$. The property "nilpotent" can be used to specify whether $B$ is a cycle generator or $\Sigma(B)=\emptyset$.

Proposition 2.4. Given a basic set $B \subseteq W_{3 ; 2}$,

(i) $B$ is a cycle generator if and only if $\mathbf{T}_{m \times n ; q}(B)$ is not nilpotent for some $m, n \geq 1$ and $0 \leq q \leq m-1$.

(ii) $\Sigma(B)=\emptyset$ if and only if $\mathbf{V}_{m \times n}(B)$ is nilpotent for some $m, n \geq 1$.

Proof. First, we have that $\mathbf{T}_{m \times n ; q}(B)$ and $\mathbf{V}_{m \times n}(B)$ are non-negative matrices. From Proposition 2.3, $B$ is easily seen to be a cycle generator if and only if $\operatorname{tr}\left(\mathbf{T}_{m \times n ; q}^{k}\right)>0$ for some $m, n, k \geq 1$ and $0 \leq q \leq m-1$. Therefore, (i) follows immediately. Similarly, from (2.3), (ii) follows. The proof is complete.

The following proposition provides an efficient method to check the nilpotent for a non-negative matrix.

Proposition 2.5. Suppose $\mathbf{A}$ is a non-negative matrix. Then, $\mathbf{A}$ is nilpotent if and only if $\mathbf{A}$ can be reduced to a zero matrix by repeating the following process: if the $i$-th row (column) of $\mathbf{A}$ is a zero row, then the $i$-th column (row) of $\mathbf{A}$ is replaced with a zero column.

Proof. Suppose the $i$-th row (column) of $\mathbf{A}$ is a zero row. Suppose $\mathbf{A}^{\prime}=\mathbf{A}$ except the $i$-th column (row), and the $i$-th column (row) of $\mathbf{A}^{\prime}$ is a zero column (row). Since $\mathbf{A}$ and 
$\mathbf{A}^{\prime}$ are non-negative,

$$
\begin{aligned}
\mathbf{A} \text { is nilpotent } & \Longleftrightarrow \operatorname{tr}\left(\mathbf{A}^{k}\right)=0 \quad \text { for all } k \geq 1 \\
& \Longleftrightarrow \operatorname{tr}\left(\mathbf{A}^{\prime k}\right)=0 \quad \text { for all } k \geq 1 \\
& \Longleftrightarrow \mathbf{A}^{\prime} \text { is nilpotent. }
\end{aligned}
$$

Therefore, from above, the result can be easily obtained. The proof is complete.

\section{Main result}

This section introduces the procedure for obtaining $\mathcal{C}(2)$ and $\mathcal{N}(2)$ by computer, and proves Wang's conjecture holds for Wang cubes with two colors.

\subsection{Periodic pairs}

This section firstly classifies the 64 Wang cubes with two colors into four groups.

For easily presenting the 64 Wang cubes, each Wang cube $w=\left(\begin{array}{c}x^{+} y^{+} z^{+} \\ x^{-} y^{-} z^{-}\end{array}\right)$is assigned a number by

$$
\varphi(w)=1+z^{-}+2 y^{-}+2^{2} x^{-}+2^{3} z^{+}+2^{4} y^{+}+2^{5} x^{+} .
$$

Definition 3.1. According to the colors on the faces in the $x$-, $y$ - and $z$-directions of a Wang cube, we classify the 64 Wang cubes into four groups.

(i) $G_{0}$ is the set of the Wang cubes that has the same color in each direction, that is, $w \in G_{0}$ if and only if

$$
w=\left(\begin{array}{lll}
a & b & c \\
a & b & c
\end{array}\right)
$$

where $a, b, c \in\{0,1\}$.

(ii) $G_{1}$ is the set of the Wang cubes that has the same colors in exactly two directions, that is, $w \in G_{1}$ if and only if

$$
w=\left(\begin{array}{lll}
a^{+} & b & c \\
a^{-} & b & c
\end{array}\right), \quad\left(\begin{array}{lll}
a & b^{+} & c \\
a & b^{-} & c
\end{array}\right) \quad \text { or } \quad\left(\begin{array}{lll}
a & b & c^{+} \\
a & b & c^{-}
\end{array}\right)
$$

where $a, b, c, a^{\beta}, b^{\beta}, c^{\beta} \in\{0,1\}, a^{-} \neq a^{+}, b^{-} \neq b^{+}$and $c^{-} \neq c^{+}$for $\beta=+,-$.

(iii) $G_{2}$ is the set of the Wang cubes that has the same color in exactly one direction, that is, $w \in G_{2}$ if and only if

$$
w=\left(\begin{array}{lll}
a^{+} & b^{+} & c \\
a^{-} & b^{-} & c
\end{array}\right),\left(\begin{array}{lll}
a^{+} & b & c^{+} \\
a^{-} & b & c^{-}
\end{array}\right) \quad \text { or } \quad\left(\begin{array}{lll}
a & b^{+} & c^{+} \\
a & b^{-} & c^{-}
\end{array}\right)
$$

where $a, b, c, a^{\beta}, b^{\beta}, c^{\beta} \in\{0,1\}, a^{-} \neq a^{+}, b^{-} \neq b^{+}$and $c^{-} \neq c^{+}$for $\beta=+,-$. 
(iv) $G_{3}$ is the set of the Wang cubes that has different colors in any direction, that is, $w \in G_{3}$ if and only if

$$
U=\left(\begin{array}{lll}
a^{+} & b^{+} & c^{+} \\
a^{-} & b^{-} & c^{-}
\end{array}\right)
$$

where $a^{\beta}, b^{\beta}, c^{\beta} \in\{0,1\}, a^{-} \neq a^{+}, b^{-} \neq b^{+}$and $c^{-} \neq c^{+}$for $\beta=+,-$.

In the following, $G_{0}, G_{1}, G_{2}$ and $G_{3}$ are listed in detail.

$$
\begin{aligned}
& G_{0}=\{1,10,19,28,37,46,55,64\} \\
& G_{1}=\{2,3,5,9,12,14,17,20,23,26,27,32,33,38,39,42,45,48,51,53,56,60,62,63\}, \\
& G_{2}=\{4,6,7,11,13,16,18,21,24,25,30,31,34,35,40,41,44,47,49,52,54,58,59,61\}, \\
& G_{3}=\{8,15,22,29,36,43,50,57\} .
\end{aligned}
$$

The following proposition shows that for each cube $w$ in $G_{i}, 0 \leq i \leq 3$, there exists a minimum cycle generator $B \subset G_{i}$ that contains $w$. The proof is omitted for brevity.

Proposition 3.2. (i) Each Wang cube $w$ in $G_{0}$ can generate a cuboid periodic pattern with period $(1,1,1)$ by repetition of itself; $\{w\}$ is then a $M C G$.

(ii) For each Wang cube $w$ in $G_{1}$, there exist exactly four Wang cubes $w_{i} \in G_{1}, 1 \leq i \leq 4$, such that $\left\{w, w_{i}\right\}$ can generate three different periodic patterns, which are cuboid periodic with period $(1,1,2),(1,2,1)$ or $(2,1,1)$, and $\left\{w, w_{i}\right\}$ is a $M C G$ for $1 \leq i \leq 4$.

(iii) For each Wang cube $w$ in $G_{2}$, there exist exactly two Wang cubes $w_{1}, w_{2} \in G_{2}$ such that $\left\{w, w_{i}\right\}$ can generate a periodic pattern, which is cuboid periodic with period $(1,2,2),(2,1,2)$ or $(2,2,1)$, and $\left\{w, w_{i}\right\}$ is a $M C G$ for $1 \leq i \leq 2$.

(iv) For each Wang cube $w$ in $G_{3}$, there exists exactly one Wang cube $w_{1} \in G_{3}$ such that $\left\{w, w_{1}\right\}$ can generate a cuboid periodic pattern with period $(2,2,2)$, and $\left\{w, w_{1}\right\}$ is a $M C G$.

Furthermore, the minimum cycle generators $B \subset G_{i}, 0 \leq i \leq 3$, can be easily found. Table A.2 presents the details.

\subsection{Algorithms}

Before the developed algorithms are presented, some notations must be introduced.

Definition 3.3. (i) For a set $A$, let $\mathbb{P}(A)$ be the power set of $A$.

(ii) For a subset $\mathcal{S} \subseteq \mathbb{P}\left(W_{3 ; 2}\right)$, let

$$
[\mathcal{S}]=\{[S] \mid S \in \mathcal{S}\}
$$


(iii) For $[B] \in\left[\mathbb{P}\left(W_{3 ; 2}\right)\right]$, let $\langle B\rangle$ be a fixed chosen element of $[B]$.

(iv) Let $\mathcal{N}^{*}(2)$ be the set of all $B \in \mathbb{P}\left(W_{3 ; 2}\right)$ such that $\Sigma(B)=\emptyset$, that is,

$$
\mathcal{N}^{*}(2)=\left\{B \in \mathbb{P}\left(W_{3 ; 2}\right) \mid \Sigma(B)=\emptyset\right\} .
$$

(v) Let $\mathcal{C}^{*}(2)$ be the set of all cycle generators, that is,

$$
\mathcal{C}^{*}(2)=\left\{B \in \mathbb{P}\left(W_{3 ; 2}\right) \mid \mathcal{P}(B) \neq \emptyset\right\}
$$

(vi) Let $\mathcal{U}^{*}(2)$ be the set of all aperiodic sets, that is,

$$
\mathcal{U}^{*}(2)=\left\{B \in \mathbb{P}\left(W_{3 ; 2}\right) \mid \mathcal{P}(B)=\emptyset, \Sigma(B) \neq \emptyset\right\}
$$

Now, the main idea of the algorithms is introduced, as follows. Let

$$
\begin{aligned}
& N=2^{64}, \quad \mathbb{P}\left(W_{3 ; 2}\right)=\left\{B_{j} \mid 0 \leq j \leq N-1\right\}, \text { where } B_{0}=\emptyset, \\
& \text { initial state for } \mathcal{C}^{*}(2): \mathcal{C}^{*}(2)=\emptyset, \\
& \text { initial state for } \mathcal{N}^{*}(2): \mathcal{N}^{*}(2)=\emptyset, \\
& \text { initial state for the set of aperiodic sets: } \mathcal{U}^{*}(2)=\emptyset .
\end{aligned}
$$

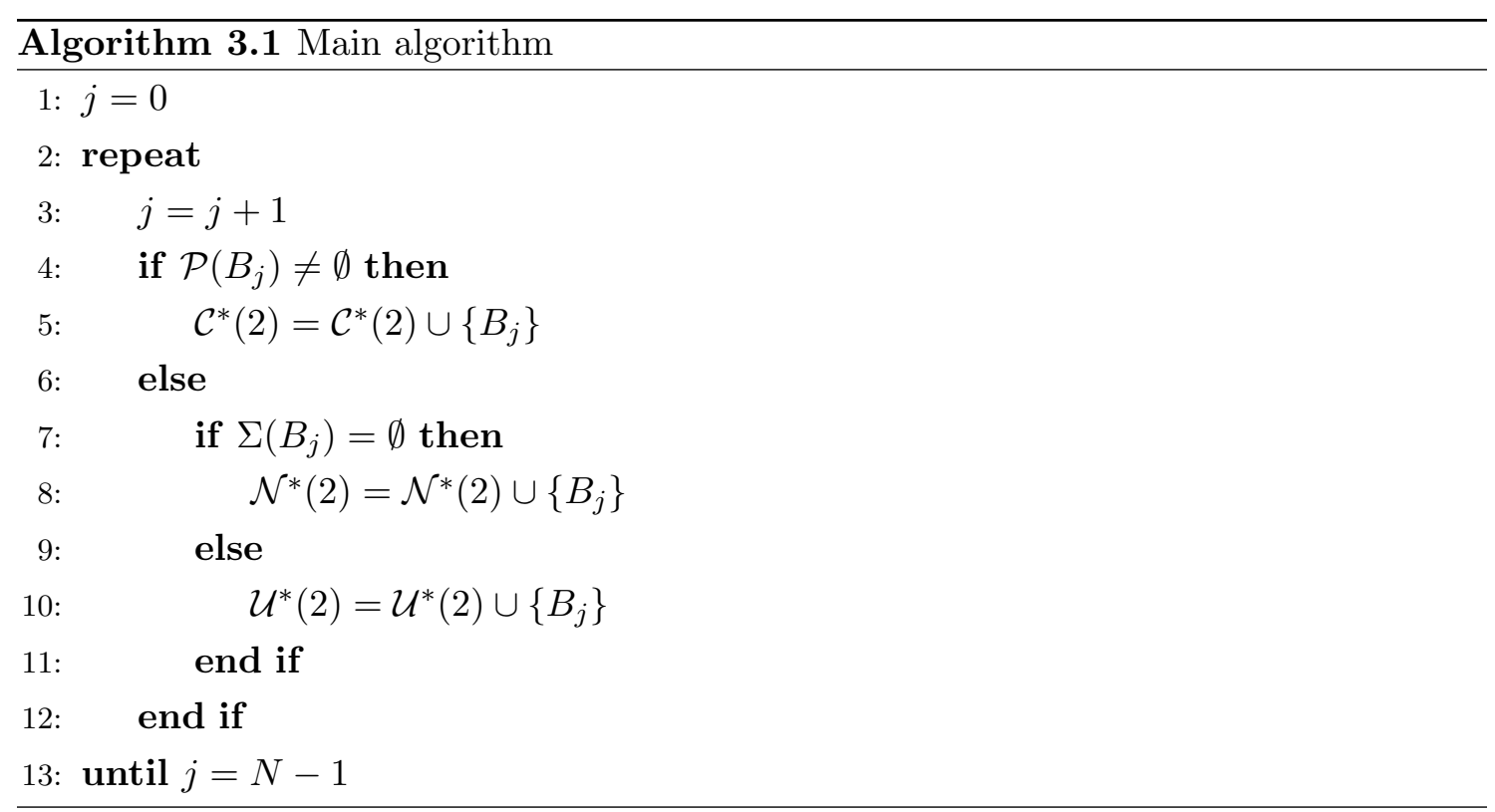

After the algorithm has been executed, if $\mathcal{U}^{*}(2)=\emptyset$ after the algorithm, then Wang's conjecture holds for Wang cubes with two colors. Moreover, if $\mathcal{U}^{*}(2)=\emptyset$, then $\mathcal{C}^{*}(2)$ is the set of all cycle generator and $\mathcal{N}^{*}(2)$ is the set of all non-cycle generators.

The methods to achieve the goal are introduced below. 
(I) reduce the number of cases that must be considered in the computation.

(II) construct efficient initial states for $\mathcal{C}^{*}(2)$ and $\mathcal{N}^{*}(2)$.

(III) construct an efficient process for determining whether or not $\mathcal{P}\left(B_{j}\right) \neq \emptyset$.

(IV) use the idea in (I), (II) and (III) to construct an efficient flowchart.

With respect to (I), the decomposition $W_{3 ; 2}=G_{0} \cup G_{1} \cup G_{2} \cup G_{3}$ is used to reduce the number of cases that must be considered in the computation. Clearly, if $B \subseteq W_{3 ; 2}$ contains a Wang cube $w \in G_{0}$, then $B$ is a cycle generator. Moreover, let $B=A_{1} \cup A_{2} \cup A_{3}$ with $A_{i} \in \mathbb{P}\left(G_{i}\right), i=1,2,3$. If $A_{i}$ is a cycle generator, then $B$ immediately satisfies (1.1). Therefore, in studying Wang's conjecture, only cases $B=A_{1} \cup A_{2} \cup A_{3}$ such that $\mathcal{P}\left(A_{i}\right)=\emptyset, i=1,2,3$, have to be considered. Table A.1 presents the details of $A_{i} \in \mathbb{P}\left(G_{i}\right)$ with $\mathcal{P}\left(A_{i}\right)=\emptyset, i=1,2,3$.

Firstly, we consider the cases $B \subseteq G_{1} \cup G_{2}$. By (2.1) and above result, the cases $B \subseteq G_{1} \cup G_{2}$ that have to be considered can be further reduced to the cases in $\mathcal{I}_{1}$ or $\mathcal{I}_{1}^{\prime}$ :

$$
\mathcal{I}_{1} \equiv\left\{A_{1} \cup\left\langle A_{2}\right\rangle \mid A_{1} \in \mathbb{P}\left(G_{1}\right) \text { and }\left[A_{2}\right] \in\left[\mathbb{P}\left(G_{2}\right)\right], \mathcal{P}\left(A_{1}\right)=\emptyset, \mathcal{P}\left(\left\langle A_{2}\right\rangle\right)=\emptyset\right\}
$$

and

$$
\mathcal{I}_{1}^{\prime} \equiv\left\{\left\langle A_{1}\right\rangle \cup A_{2} \mid\left[A_{1}\right] \in\left[\mathbb{P}\left(G_{1}\right)\right] \text { and } A_{2} \in \mathbb{P}\left(G_{2}\right), \mathcal{P}\left(\left\langle A_{1}\right\rangle\right)=\emptyset, \mathcal{P}\left(A_{2}\right)=\emptyset\right\}
$$

From Table A.1. $\left|\mathcal{I}_{1}\right|=1.120104 \times 10^{7}$ and $\left|\mathcal{I}_{1}^{\prime}\right|=9.078498 \times 10^{6}$. Therefore, $\mathcal{I}_{1}^{\prime}$ is the better choice for reducing $B \subseteq G_{1} \cup G_{2}$. Notably, $\left|\mathcal{I}_{1}^{\prime}\right| \ll\left|\mathbb{P}\left(G_{1} \cup G_{2}\right)\right|=2^{48} \approx 2.8417 \times 10^{14}$; the reduction is considerable. Indeed, for $A_{i} \in \mathbb{P}\left(G_{i}\right), 1 \leq i \leq 3$, we verify that $\mathcal{P}(B)=\emptyset$ implies $\Sigma(B)=\emptyset$ by computer. Moreover, for $A_{i} \in \mathbb{P}\left(G_{i}\right), 1 \leq i \leq 3, A_{i}$ satisfies (1.1).

Next, it is clear that if $B$ contains a cycle generator of $G_{1} \cup G_{2}$, then $B$ satisfies (1.1). Hence, we only consider the cases $B \subseteq N\left(G_{1} \cup G_{2}\right) \cup G_{3}$, where $N\left(G_{1} \cup G_{2}\right)$ is the set of non-cycle generators in $\mathbb{P}\left(G_{1} \cup G_{2}\right)$. Similarly, the cases $B \subseteq N\left(G_{1} \cup G_{2}\right) \cup G_{3}$ that have to be considered can be further reduced as 3.1 and 3.2 .

With respect to $(\mathrm{II})$, the initial state for $\mathcal{C}^{*}(2)$ are given by the set of all minimal cycle generators that are the subsets of $G_{i}, 1 \leq i \leq 3 . \mathcal{C}^{*}(2)$ and $\mathcal{N}^{*}(2)$ can be easily found using a computer program. See Tables A.2 and A.5.

With respect to (III), using the generalized trace operators $\mathbf{T}_{m \times n ; q}, 0 \leq q \leq 1$, to find a periodic pattern is more efficient than only using the trace operator $\mathbf{T}_{m \times n}$. The following example illustrates this idea. 
Example 3.4. (a) Let

$$
\begin{aligned}
B_{1} & =\{2,7,20,43,61\} \\
& =\left\{\left(\begin{array}{lll}
0 & 0 & 0 \\
0 & 0 & 1
\end{array}\right),\left(\begin{array}{lll}
0 & 0 & 0 \\
1 & 1 & 0
\end{array}\right),\left(\begin{array}{lll}
0 & 1 & 0 \\
0 & 1 & 1
\end{array}\right),\left(\begin{array}{lll}
1 & 0 & 1 \\
0 & 1 & 0
\end{array}\right),\left(\begin{array}{lll}
1 & 1 & 1 \\
1 & 0 & 0
\end{array}\right)\right\} .
\end{aligned}
$$

By computer, it can be verified that $B_{1}$ is a MCG, and it can generate a cuboid periodic pattern $U_{1}$ with period $(7,14,14)$. Using Propositions 2.4 and 2.5 to find this periodic pattern, we need to show that the trace operator $\mathbf{T}_{7 \times 14}\left(B_{1}\right)$ is not nilpotent. However, the size of $\mathbf{T}_{7 \times 14}\left(B_{1}\right)$ is $2^{7 \cdot 14} \times 2^{7 \cdot 14}$, and it costs too much memories of computer. Considering parallelopiped period, $U_{1}$ can be represented as a $\left[\begin{array}{lll}7 & 3 & 0 \\ 0 & 2 & 0 \\ 0 & 0 & 14\end{array}\right]$-periodic pattern. Then, the associated generalized trace operator is $\mathbf{T}_{7 \times 2 ; 3}\left(B_{1}\right)$ with size $2^{7 \cdot 2} \times 2^{7 \cdot 2}$, which is much less than that of $\mathbf{T}_{7 \times 14}\left(B_{1}\right)$. Therefore, in general, generalized trace operators provide a more efficient method to determine $\mathcal{P}(B) \neq \emptyset$.

(b) Let

$$
\begin{aligned}
B_{2} & =\{6,7,25,36,61\} \\
& =\left\{\left(\begin{array}{lll}
0 & 0 & 0 \\
1 & 0 & 1
\end{array}\right),\left(\begin{array}{lll}
0 & 0 & 0 \\
1 & 1 & 0
\end{array}\right),\left(\begin{array}{lll}
0 & 1 & 1 \\
0 & 0 & 0
\end{array}\right),\left(\begin{array}{lll}
1 & 0 & 0 \\
0 & 1 & 1
\end{array}\right),\left(\begin{array}{lll}
1 & 1 & 1 \\
1 & 0 & 0
\end{array}\right)\right\} \\
& \in[\{4,6,15,49,58\}] .
\end{aligned}
$$

It can be shown that $B_{2}$ is a MCG, and it can generate a cuboid periodic pattern $U_{2}$ with period $(7,28,28)$. In fact, $U_{2}$ can be represented as a $\left[\begin{array}{lll}7 & 3 & 0 \\ 0 & 4 & 0 \\ 0 & 0 & 28\end{array}\right]$-periodic pattern. Similar to (a), it is easier to find $U_{2}$ using $\mathbf{T}_{7 \times 4 ; 3}\left(B_{2}\right)$ than $\mathbf{T}_{7 \times 28}\left(B_{2}\right)$.

(c) Let

$$
\begin{aligned}
B_{3} & =\{2,7,29,48,51\} \\
& =\left\{\left(\begin{array}{lll}
0 & 0 & 0 \\
0 & 0 & 1
\end{array}\right),\left(\begin{array}{lll}
0 & 0 & 0 \\
1 & 1 & 0
\end{array}\right),\left(\begin{array}{lll}
0 & 1 & 1 \\
1 & 0 & 0
\end{array}\right),\left(\begin{array}{lll}
1 & 0 & 1 \\
1 & 1 & 1
\end{array}\right),\left(\begin{array}{lll}
1 & 1 & 0 \\
0 & 1 & 0
\end{array}\right)\right\} .
\end{aligned}
$$

We have that $B_{3}$ is a MCG, and it can generate a cuboid periodic pattern $U_{3}$ with period $(14,14,14)$. In fact, $U_{3}$ can be represented as a $\left[\begin{array}{ccc}14 & 3 & 0 \\ 0 & 1 & 0 \\ 0 & 0 & 14\end{array}\right]$-periodic pattern. So, by checking that $\mathbf{T}_{14 \times 1 ; 3}\left(B_{3}\right)$ is not nilpotent, $U_{3}$ can be found.

Furthermore, the periodical patterns $U_{1}, U_{2}$ and $U_{3}$ are clearly described in Remark 3.7 (iii).

With respect to (IV), the flowchart, which is based on (I), (II) and (III), is as follows. 


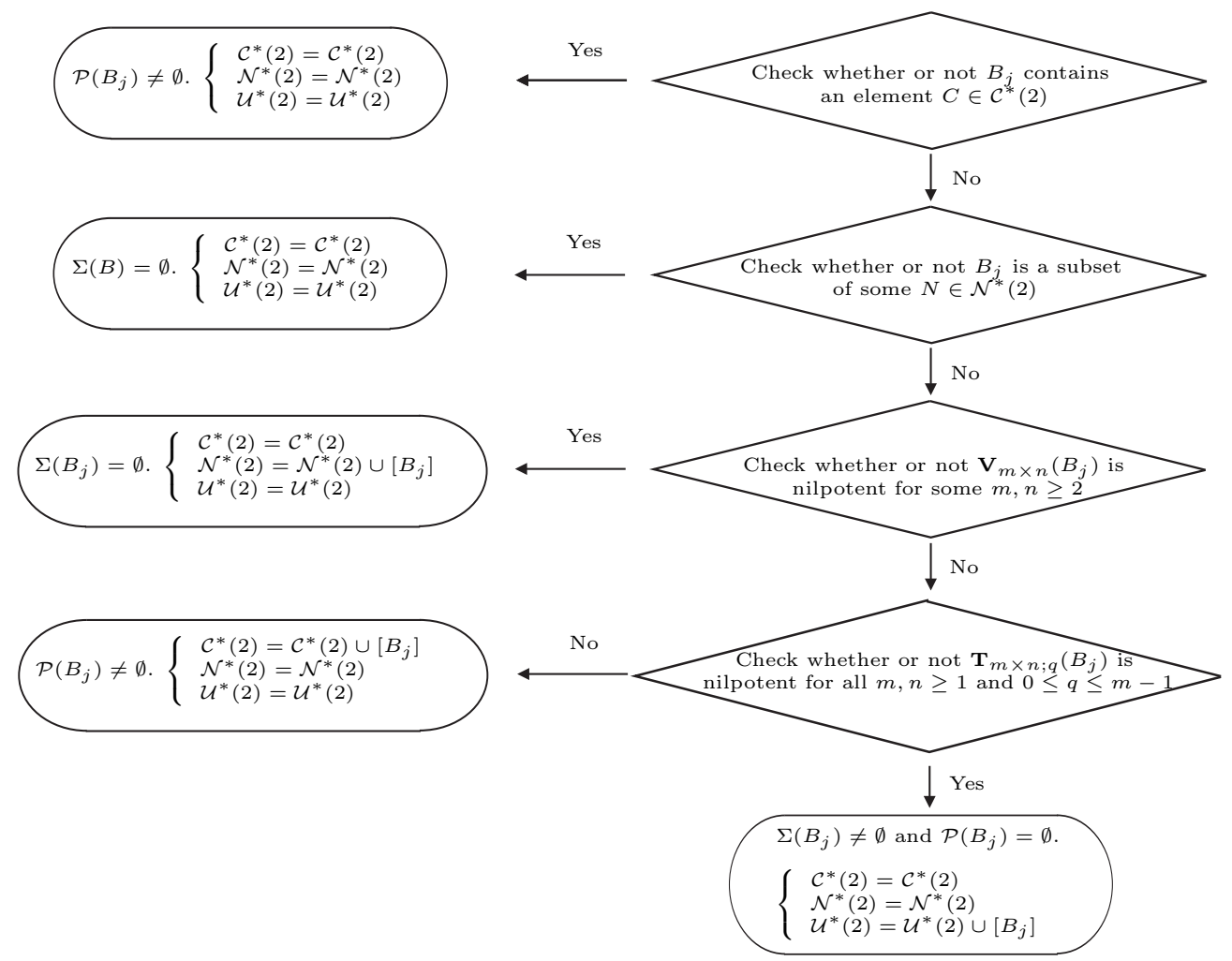

Figure 3.1: Flowchart.

In real computation, we use the above flowchart twice to complete our algorithm, as follows.

Step 1. The cases $B \in \mathcal{I}_{1}^{\prime}$ defined in 3.2 are considered. Set

$$
\begin{aligned}
\mathcal{C}^{*}(2) & \equiv\left\{B \in \mathbb{P}\left(G_{i}\right) \text { for } i=0,1,2,3 \mid \mathcal{P}(B) \neq \emptyset\right\}, \\
\mathcal{N}^{*}(2) & \equiv\left\{B \in \mathbb{P}\left(G_{i}\right) \text { for } i=1,2 \mid \Sigma(B)=\emptyset\right\}, \\
\mathcal{U}^{*}(2) & \equiv\{\emptyset\}
\end{aligned}
$$

as the initial state of the flowchart. After the flowchart, we get three new sets: $\mathcal{C}^{*}(2)$, $\mathcal{N}^{*}(2)$ and $\mathcal{U}^{*}(2)$.

Step 2. Consider the cases

$$
B \in \mathcal{I}_{2} \equiv\left\{\langle A\rangle \cup A_{3} \mid[A] \in\left[\mathbb{P}\left(\mathcal{N}^{*}(2)\right)\right] \text {, and } A_{3} \in \mathbb{P}\left(G_{3}\right), \mathcal{P}\left(A_{3}\right)=\emptyset\right\},
$$

where $\mathcal{N}^{*}(2)$ in $\mathcal{I}_{2}$ is obtained from Step 1. Notably, for $A \in \mathbb{P}\left(\mathcal{N}^{*}(2)\right)$, we have $\Sigma(A)=\emptyset$, 
which implies $\mathcal{P}(A)=\emptyset$. In addition, the initial states $\mathcal{C}^{*}(2), \mathcal{N}^{*}(2)$ and $\mathcal{U}^{*}(2)$ are the results from Step 1.

Remark 3.5. Suppose that the computation based on the flowchart has been completed.

(i) If the set $\mathcal{U}^{*}(2)=\emptyset$, then Wang's conjecture holds for Wang cubes with two colors; otherwise, every element in $\mathcal{U}^{*}(2)$ is an aperiodic set.

(ii) It is easy to see that an element in $\mathcal{C}^{*}(2)$ may be not a minimal cycle generator. However, $\mathcal{C}(2)$ can be obtained from $\mathcal{C}^{*}(2)$ by the following process: First, set $\mathcal{C}(2)$ equals to $\mathcal{C}^{*}(2)$. If $C_{1}, C_{2} \in \mathcal{C}(2)$ with $C_{1} \subsetneq C_{2}$, then $C_{2}$ must be removed from $\mathcal{C}(2)$. Indeed,

$$
\mathcal{C}(2)=\left\{B \in \mathcal{C}^{*}(2) \mid B \text { doesn't contain any } B^{\prime} \in \mathcal{C}^{*}(2) \text { except itself }\right\}
$$

(iii) In a manner similar to that for (ii), we can obtain $\mathcal{N}(2)$ from $\mathcal{N}^{*}(2)$. Indeed,

$$
\begin{aligned}
\mathcal{N}(2)=\left\{B \in \mathcal{N}^{*}(2) \mid\right. & B \text { is not a proper subset of } B^{\prime} \\
& \text { for all } \left.B^{\prime} \in \mathcal{N}^{*}(2) \text { except itself }\right\} .
\end{aligned}
$$

\subsection{Main result}

The computer program of Figure 3.1 is written, and the computation is completed in finite time. These cases can be computed completely within a week. The main result is as follows.

Theorem 3.6. The set $\mathcal{U}^{*}(2)=\emptyset$, and then Wang's conjecture holds for Wang cubes with two colors.

Remark 3.7. (i) $\mathcal{C}(2)$ and $\mathcal{N}(2)$ can be obtained and their numbers are listed in Table A.5.

(ii) From the computational results, if $\mathcal{B} \subseteq W_{3 ; 2}$ is a cycle generator, then there exists $B^{\prime} \in[B]$ such that $\mathbf{T}_{m \times n}\left(B^{\prime}\right)$ is not nilpotent for some $1 \leq m \leq 14$ and $1 \leq n \leq 28$; see Tables A.2 A.4 for details. Among them, the most complicated cases are $B_{1}, B_{2}$ and $B_{3}$ in Example 3.4, they can be verified that they are cycle generators by checking $\mathbf{T}_{7 \times 2 ; 3}\left(B_{1}\right)$, $\mathbf{T}_{7 \times 4 ; 3}\left(B_{2}\right)$ and $\mathbf{T}_{14 \times 1 ; 3}\left(B_{3}\right)$ are not nilpotent, respectively.

On the other hand, from the computational results, if $\Sigma(\mathcal{B})=\emptyset$ for $\mathcal{B} \subseteq W_{3 ; 2}$, then there exists $B^{\prime} \in[B]$ such that $\mathbf{V}_{m \times n}\left(B^{\prime}\right)$ is nilpotent for some $1 \leq m \leq 4$ and $1 \leq n \leq 3$. For example, consider

$$
\begin{aligned}
B_{4} & =\{2,7,15,20,41,62\} \\
& =\left\{\left(\begin{array}{lll}
0 & 0 & 0 \\
0 & 0 & 1
\end{array}\right),\left(\begin{array}{lll}
0 & 0 & 0 \\
1 & 1 & 0
\end{array}\right),\left(\begin{array}{lll}
0 & 1 & 1 \\
1 & 0 & 0
\end{array}\right),\left(\begin{array}{lll}
1 & 0 & 1 \\
1 & 1 & 1
\end{array}\right),\left(\begin{array}{lll}
1 & 1 & 0 \\
0 & 1 & 0
\end{array}\right),\left(\begin{array}{lll}
1 & 1 & 0 \\
0 & 1 & 0
\end{array}\right)\right\} .
\end{aligned}
$$


$\mathbf{V}_{4 \times 3}\left(B_{4}\right)$ is nilpotent, and $\mathbf{V}_{m \times n}\left(B_{4}\right)$ are not nilpotent for all $1 \leq m \leq 3$ and $1 \leq n \leq 2$.

(iii) (a) Continue to study $B_{1}$ and $U_{1}=\left(u_{1 ; i_{1}, i_{2}, i_{3}}\right)$ in Example 3.4(a). Through more careful verification by computer, $U_{1}$ is a $\left[\begin{array}{lll}7 & 3 & 1 \\ 0 & 2 & 1 \\ 0 & 0 & 1\end{array}\right]$-periodic pattern with

$$
\begin{aligned}
& {\left[\begin{array}{lllllll}
u_{1 ; 0,1,0} & u_{1 ; 1,1,0} & u_{1 ; 2,1,0} & u_{1 ; 3,1,0} & u_{1 ; 4,1,0} & u_{1 ; 5,1,0} & u_{1 ; 6,1,0} \\
u_{1 ; 0,0,0} & u_{1 ; 1,0,0} & u_{1 ; 2,0,0} & u_{1 ; 3,0,0} & u_{1 ; 4,0,0} & u_{1 ; 5,0,0} & u_{1 ; 6,0,0}
\end{array}\right] } \\
= & {\left[\begin{array}{ccccccc}
61 & 61 & 7 & 2 & 20 & 43 & 61 \\
2 & 43 & 61 & 7 & 20 & 20 & 2
\end{array}\right] . }
\end{aligned}
$$

Therefore, by (1.3), the periodic pattern $U_{1}$ is understood completely.

(b) Consider $B_{2}$ and $U_{2}=\left(u_{2 ; i_{1}, i_{2}, i_{3}}\right)$ in Example $3.4(\mathrm{~b})$. It can be verified that $U_{2}$ is $\mathrm{a}\left[\begin{array}{lll}7 & 3 & 0 \\ 0 & 4 & 1 \\ 0 & 0 & 1\end{array}\right]$-periodic pattern with

$$
\begin{aligned}
& {\left[\begin{array}{lllllll}
u_{2 ; 0,3,0} & u_{2 ; 1,3,0} & u_{2 ; 2,3,0} & u_{2 ; 3,3,0} & u_{2 ; 4,3,0} & u_{2 ; 5,3,0} & u_{2 ; 6,3,0} \\
u_{2 ; 0,2,0} & u_{2 ; 1,2,0} & u_{2 ; 2,2,0} & u_{2 ; 3,2,0} & u_{2 ; 4,2,0} & u_{2 ; 5,2,0} & u_{2 ; 6,2,0} \\
u_{2 ; 0,1,0} & u_{2 ; 1,1,0} & u_{2 ; 2,1,0} & u_{2 ; 3,1,0} & u_{2 ; 4,1,0} & u_{2 ; 5,1,0} & u_{2 ; 6,1,0} \\
u_{2 ; 0,0,0} & u_{2 ; 1,0,0} & u_{2 ; 2,0,0} & u_{2 ; 3,0,0} & u_{2 ; 4,0,0} & u_{2 ; 5,0,0} & u_{2 ; 6,0,0}
\end{array}\right]} \\
& =\left[\begin{array}{ccccccc}
36 & 6 & 25 & 36 & 7 & 25 & 25 \\
61 & 7 & 36 & 61 & 61 & 6 & 36 \\
36 & 61 & 61 & 6 & 36 & 7 & 25 \\
61 & 6 & 36 & 7 & 25 & 25 & 36
\end{array}\right] .
\end{aligned}
$$

So, by 1.3 , the periodic pattern $U_{2}$ is all now clear.

(c) For $B_{3}$ and $U_{3}=\left(u_{3 ; i_{1}, i_{2}, i_{3}}\right)$ in Example 3.4(c), after further study, $U_{3}$ can be represented as a $\left[\begin{array}{ccc}14 & 3 & 5 \\ 0 & 1 & 0 \\ 0 & 0 & 1\end{array}\right]$-periodic pattern, and the Wang cubes in $u_{3 ; i_{1}, 0,0}$, where $i_{1}$ goes from 0 to 13 , are

$$
2 \rightarrow 51 \rightarrow 29 \rightarrow 2 \rightarrow 51 \rightarrow 48 \rightarrow 7 \rightarrow 51 \rightarrow 29 \rightarrow 51 \rightarrow 29 \rightarrow 2 \rightarrow 51 \rightarrow 7
$$

By (1.3), the periodic pattern $U_{3}$ is understood completely.

For completeness, Table A.5 gives the numbers of minimal cycle generators and maximal non-cycle generators.

\section{A. Appendices}

Table A.1 presents the numbers of equivalence classes of non-cycle generators in $G_{i}$ with two colors. Only the non-zero cases are listed. 


\begin{tabular}{|l|c|c|c|c|c|c|}
\hline Number of cubes & {$\left[G_{1}\right]$} & $G_{1}$ & {$\left[G_{2}\right]$} & $G_{2}$ & {$\left[G_{3}\right]$} & $G_{3}$ \\
\hline 1 cube & 1 & 24 & 1 & 24 & 1 & 8 \\
\hline 2 cubes & 4 & 228 & 5 & 252 & 2 & 24 \\
\hline 3 cubes & 9 & 1112 & 12 & 1456 & 2 & 32 \\
\hline 4 cubes & 18 & 3126 & 34 & 4860 & 2 & 14 \\
\hline 5 cubes & 24 & 5664 & 54 & 10320 & & \\
\hline 6 cubes & 30 & 7056 & 80 & 14792 & & \\
\hline 7 cubes & 24 & 6240 & 74 & 14736 & & \\
\hline 8 cubes & 18 & 3948 & 62 & 10266 & & \\
\hline 9 cubes & 9 & 1760 & 33 & 4920 & & \\
\hline 10 cubes & 4 & 528 & 16 & 1548 & & \\
\hline 11 cubes & 1 & 96 & 4 & 288 & & \\
\hline 12 cubes & 1 & 8 & 1 & 24 & & \\
\hline Total: & 143 & 29790 & 376 & 63486 & 7 & 78 \\
\hline
\end{tabular}

Table A.1

Tables A.2, A.3 and A.4 present the equivalence classes of the minimal cycle generators with two colors. The symbol $[B]_{(m, n, k)}$ presents one of the equivalence classes $[B]$ with the periodic $(m, n, k)$ which satisfy $m \leq n \leq k$.

\begin{tabular}{|c|ll|}
\hline$k$ & {$[C] \in\left[\mathcal{C}\left(G_{0}\right)\right]$ with $k$ cubes } & \\
\hline 1 & {$[\{1\}]_{(1,1,1)}$} & \\
\hline \hline$k$ & {$[C] \in\left[\mathcal{C}\left(G_{1}\right)\right]$ with $k$ cubes } & \\
\hline 2 & {$[\{2,9\}]_{(1,1,2)}$} & {$[\{2,27\}]_{(1,1,2)}$} \\
\hline \hline$k$ & {$[C] \in\left[\mathcal{C}\left(G_{2}\right)\right]$ with $k$ cubes } & \\
\hline 2 & {$[\{4,25\}]_{(1,2,2)}$} & {$[\{4,61\}]_{(1,2,2)}$} \\
\hline 3 & {$[\{4,13,49\}]_{(3,3,3)}$} & \\
\hline 4 & {$[\{4,11,21,49\}]_{(2,2,2)}$} & {$[\{4,11,21,58\}]_{(2,2,2)}$} \\
\hline \hline$k$ & {$[C] \in\left[\mathcal{C}\left(G_{3}\right)\right]$ with $k$ cubes } & \\
\hline 2 & {$[\{8,57\}]_{(2,2,2)}$} & \\
\hline 4 & {$[\{8,29,43,50\}]_{(2,2,2)}$} & \\
\hline
\end{tabular}

Table A.2 


\begin{tabular}{|c|lll|}
\hline$k$ & {$[C] \in\left[\mathcal{C}\left(G_{1} G_{2}\right)\right]$ with $k$ cubes } & & \\
\hline \multirow{3}{*}{3} & {$[\{2,3,25\}]_{(1,3,3)}$} & {$[\{2,3,61\}]_{(1,3,3)}$} & {$[\{2,11,25\}]_{(1,2,2)}$} \\
& {$[\{2,11,53\}]_{(1,3,3)}$} & {$[\{2,11,61\}]_{(1,2,2)}$} & {$[\{2,31,59\}]_{(1,2,2)}$} \\
\hline \multirow{3}{*}{4} & {$[\{2,3,13,49\}]_{(2,2,2)}$} & {$[\{2,3,13,58\}]_{(2,4,4)}$} & {$[\{2,3,30,59\}]_{(4,4,4)}$} \\
& {$[\{2,7,21,41\}]_{(2,3,3)}$} & {$[\{2,7,21,59\}]_{(2,3,3)}$} & {$[\{2,7,25,41\}]_{(5,5,5)}$} \\
& {$[\{2,7,25,44\}]_{(2,3,6)}$} & {$[\{2,7,25,51\}]_{(4,4,4)}$} & {$[\{2,7,25,59\}]_{(5,5,5)}$} \\
\hline 5 & {$[\{2,7,20,44,61\}]_{(2,2,3)}$} & & \\
\hline 6 & {$[\{2,4,7,41,59,62\}]_{(3,3,3)}$} & {$[\{2,7,16,51,60,61\}]_{(3,3,4)}$} & \\
\hline
\end{tabular}

Table A.3

\begin{tabular}{|l|lll|}
\hline$k$ & {$[C] \in\left[\mathcal{C}\left(G_{1} G_{2} G_{3}\right)\right]_{\text {with } k \text { cubes }}$} & & \\
\hline 3 & {$[\{2,7,57\}]_{(3,3,3)}$} & {$[\{2,15,57\}]_{(2,2,2)}$} & {$[\{4,29,57\}]_{(2,2,2)}$} \\
\hline \multirow{2}{*}{4} & {$[\{2,3,5,57\}]_{(2,2,2)}$} & {$[\{2,3,13,57\}]_{(5,5,5)}$} & {$[\{2,3,14,57\}]_{(4,4,4)}$} \\
& {$[\{2,3,29,57\}]_{(2,3,3)}$} & {$[\{2,3,29,58\}]_{(5,5,5)}$} & {$[\{2,4,13,57\}]_{(2,2,2)}$} \\
& {$[\{2,4,29,59\}]_{(2,4,4)}$} & {$[\{2,8,25,43\}]_{(2,3,6)}$} & {$[\{2,8,25,59\}]_{(2,4,4)}$} \\
& {$[\{2,8,25,41\}]_{(2,2,2)}$} & {$[\{2,11,24,57\}]_{(2,4,4)}$} & {$[\{2,11,29,57\}]_{(2,2,2)}$} \\
& {$[\{2,11,13,57\}]_{(2,3,3)}$} & {$[\{2,15,29,51\}]_{(2,3,3)}$} & {$[\{2,15,29,59\}]_{(2,2,2)}$} \\
& {$[\{2,11,29,59\}]_{(2,3,3)}$} & {$[\{4,6,11,57\}]_{(2,3,3)}$} & {$[\{4,11,21,50\}]_{(2,4,8)}$} \\
& {$[\{2,15,59,61\}]_{(2,3,3)}$} & {$[\{4,13,50,57\}]_{(3,3,2)}$} & {$[\{4,15,54,57\}]_{(2,2,2)}$} \\
& {$[\{4,11,22,57\}]_{(2,2,2)}$} & {$[\{2,4,15,53,59\}]_{(2,3,3)}$} & {$[\{2,4,21,47,57\}]_{(2,5,5)}$} \\
\hline & {$[\{2,3,29,48,60\}]_{(2,4,4)}$} & {$[\{2,7,20,43,53\}]_{(5,10,10)}$} & {$[\{2,7,20,43,61\}]_{(7,14,14)}$} \\
& {$[\{2,7,16,29,51\}]_{(2,5,5)}$} & {$[\{2,7,50,59,61\}]_{(3,3,6)}$} & {$[\{2,8,20,43,61\}]_{(2,2,3)}$} \\
& {$[\{2,7,29,48,51\}]_{(14,14,14)}$} & {$[\{4,6,15,24,57\}]_{(2,2,4)}$} & {$[\{4,6,15,49,57\}]_{(2,5,5)}$} \\
5 & {$[\{2,8,29,54,59\}]_{(2,8,8)}$} & {$[\{4,6,24,47,57\}]_{(2,2,3)}$} & {$[\{4,6,29,43,49\}]_{(5,5,5)}$} \\
& {$[\{4,6,15,49,58\}]_{(7,28,28)}$} & {$[\{4,8,29,41,50\}]_{(2,2,2)}$} & {$[\{4,8,29,41,54\}]_{(2,3,3)}$} \\
& {$[\{4,6,29,43,50\}]_{(2,6,6)}$} & {$[\{4,13,22,50,59\}]_{(2,2,2)}$} & {$[\{4,15,22,40,57\}]_{(2,2,3)}$} \\
\hline & {$[\{4,13,22,40,57\}]_{(2,2,5)}$} & {$[\{2,3,11,32,38,57\}]_{(3,9,9)}$} & {$[\{2,3,29,38,39,60\}]_{(3,3,4)}$} \\
& {$[\{2,3,6,29,39,60\}]_{(3,3,3)}$} & {$[\{2,7,22,44,59,61\}]_{(3,3,6)}$} & {$[\{2,7,29,47,50,60\}]_{(3,3,6)}$} \\
& {$[\{2,7,15,51,60,61\}]_{(3,3,3)}$} & {$[\{4,6,15,24,49,58\}]_{(2,6,7)}$} & \\
\hline
\end{tabular}

Table A.4 
Table A.5 shows the numbers of $\mathcal{C}(2)$ and $\mathcal{N}(2)$. Firstly, denote by

$$
\begin{aligned}
\mathcal{C}_{k}(2) & =\{B \in \mathcal{C}(2):|B|=k\}, \\
\mathcal{N}_{k}(2) & =\{B \in \mathcal{N}(2):|B|=k\}, \\
{\left[\mathcal{C}_{k}(2)\right] } & =\left\{[B] \in[\mathcal{C}(2)]:\left|B^{\prime}\right|=k \text { for all } B^{\prime} \in[B]\right\}, \\
{\left[\mathcal{N}_{k}(2)\right] } & =\left\{[B] \in[\mathcal{N}(2)]:\left|B^{\prime}\right|=k \text { for all } B^{\prime} \in[B]\right\} .
\end{aligned}
$$

Clearly, from Proposition $3.2, \mathcal{C}(2)=\bigcup_{k=1}^{28} \mathcal{C}_{k}(2)$ and $\mathcal{N}(2)=\bigcup_{k=1}^{28} \mathcal{N}_{k}(2)$. Only the cases for $\mathcal{C}_{k}(2) \neq \emptyset$ and $\mathcal{N}_{k}(2) \neq \emptyset$ are listed.

\begin{tabular}{|c|c|c|c|c|}
\hline$k$ & $\#\left\{\mathcal{C}_{k}(2)\right\}$ & $\#\left\{\left[\mathcal{C}_{k}(2)\right]\right\}$ & $\#\left\{\mathcal{N}_{k}(2)\right\}$ & $\#\left\{\left[\mathcal{N}_{k}(2)\right]\right\}$ \\
\hline 1 & 8 & 1 & & \\
\hline 2 & 76 & 6 & & \\
\hline 3 & 904 & 10 & & \\
\hline 4 & 6042 & 37 & & \\
\hline 5 & 4056 & 22 & & \\
\hline 6 & 2544 & 10 & 2688 & 7 \\
\hline 7 & & & 3072 & 9 \\
\hline 8 & & & 48 & 1 \\
\hline 9 & & & 1824 & 7 \\
\hline 10 & & & 96 & 1 \\
\hline 11 & & & 192 & 1 \\
\hline 12 & & & 0 & 0 \\
\hline$\vdots$ & & & $\vdots$ & $\vdots$ \\
\hline 27 & & & 0 & 0 \\
\hline 28 & & & 96 & 2 \\
\hline
\end{tabular}

Table A.5

\section{References}

[1] J.-C. Ban, W.-G. Hu, S.-S. Lin and Y.-H. Lin, Zeta functions for two-dimensional shifts of finite type, Mem. Amer. Math. Soc. 221 (2013), no. 1037, 60 pp.

[2] J.-C. Ban, S.-S. Lin and Y.-H. Lin, Patterns generation and spatial entropy in twodimensional lattice models, Asian J. Math. 11 (2007), no. 3, 497-534. 
[3] R. J. Baxter, Eight-vertex model in lattice statistics, Phys. Rev. Lett. 26 (1971), no. $14,832-833$.

[4] _ Exactly Solved Models in Statistical Mechanics, Academic Press, London, 1982.

[5] R. Berger, The undecidability of the domino problem, Mem. Amer. Math. Soc. 66 (1966), $72 \mathrm{pp}$.

[6] H.-H. Chen, W.-G. Hu, D.-J. Lai and S.-S. Lin, Nonemptiness problems of Wang tiles with three colors, Theoret. Comput. Sci. 547 (2014), 34-45.

[7] J.-Y. Chen, Y.-J. Chen, W.-G. Hu and S.-S. Lin, Spatial chaos of Wang tiles with two symbols, J. Math. Phys. 57 (2015), no. 2, 022704, 13 pp.

[8] K. Culik II, An aperiodic set of 13 Wang tiles, Discrete Math. 160 (1996), no. 1-3, $245-251$.

[9] K. Culik II and J. Kari, An aperiodic set of Wang cubes, J.UCS 1 (1995), no. 10, $675-686$.

[10] B. Grünbaum and G. C. Shephard, Tilings and Patterns, New York, 1987.

[11] W.-G. Hu and S.-S. Lin, Zeta functions for higher-dimensional shifts of finite type, Internat. J. Bifur. Chaos Appl. Sci. Engrg. 19 (2009), no. 11, 3671-3689.

[12] _ Nonemptiness problems of plane square tiling with two colors, Proc. Amer. Math. Soc. 139 (2011), no. 3, 1045-1059.

[13] E. Jeandel and M. Rao, An aperiodic set of 11 Wang tiles, arXiv:1506.06492.

[14] J. Kari, A small aperiodic set of Wang tiles, Discrete Math. 160 (1996), no. 1-3, $259-264$.

[15] A. Lagae, J. Kari and P. Dutré, Aperiodic sets of square tiles with colored corners, Report CW 460, Department of Computer Science, Katholieke Universiteit Leuven, Belgium, August 2006.

[16] E. H. Lieb, Exact solution of the problem of the entropy of two-dimensional ice, Phys. Rev. Lett. 18 (1967), no. 17, 692-694.

[17] _ Residual entropy of square ice, Phys. Rev. 162 (1967), no. 1, 162-172.

[18] R. Penrose, The role of aesthetics in pure and applied mathematical research, Bull. Inst. Math. Appl. 10 (1974), 266-271. 
[19] R. M. Robinson, Undecidability and nonperiodicity for tilings of the plane, Invent. Math. 12 (1971), 177-209.

[20] H. Wang, Proving theorems by pattern recognition - II, Bell System Tech. J. 40 (1961), no. $1,1-41$.

Hung-Hsun Chen

Department of Applied Mathematics, National Chiao Tung University, Hsinchu 300, Taiwan

E-mail address: hhchen@nctu.edu.tw

Wen-Guei $\mathrm{Hu}$

College of Mathematics, Sichuan University, Chengdu, 610064, China

E-mail address: wghu@scu.edu.cn

Song-Sun Lin

Department of Applied Mathematics, National Chiao Tung University, Hsinchu 30010, Taiwan

E-mail address: sslin@math.nctu.edu.tw 